ORIGINAL ARTICLE

\title{
Effect of off-road competitive motocross race on plasma oxidative stress and damage markers
}

\author{
António Ascensão, Rita Ferreira, Franklim Marques, Eduardo Oliveira, Victor Azevedo, José Soares, \\ José Magalhães
}

Br J Sports Med 2007;41:101-105. doi: 10.1136/bjsm.2006.031591

\begin{abstract}
See end of article for authors' affiliations

.....................

Correspondence to: Professor A Ascensão, Research Center in Physical Activity, Health and Leisure, Faculty of Sports Sciences, University of Porto, Porto 4200-450, Portugal: aascensao@fcdef.up.pt
\end{abstract}

Accepted 8 November 2006 Published Online First 29 November 2006

\begin{abstract}
Aim: To analyse the effect of an off-road motocross heat on plasma levels of oxidative stress and damage, blood leucocyte counts and urine catecholamine concentration.

Methods: Plasma contents of total, reduced and oxidised (GSSG) glutathione, \%GSSG, malondialdehyde (MDA), protein carbonyl and sulphydryl groups, total antioxidant status (TAS), uric acid, and blood neutrophil and lymphocyte counts were evaluated in 10 male top-level riders before, immediately after $(0 \mathrm{~h})$ and $1 \mathrm{~h}$ after a simulated competitive motocross race. 24-h urine adrenaline, noradrenaline and dopamine concentrations were also measured.

Results: The motocross heat resulted in an increase in plasma oxidative stress and damage $(p<0.05)$. This was shown by a significant increase in \%GSSG, TAS, MDA and carbonyls, and by a decrease in sulphydryl groups after the race. There was a significant increase in both plasma uric acid and urine catecholamine concentration after the race $(p<0.05)$. Blood neutrophil counts increased at 0 and $1 \mathrm{~h}$ after exercise $(\mathrm{p}<0.05)$. Lymphocyte count increased from baseline to $0 \mathrm{~h}$, although it decreased from baseline and 0 to $1 \mathrm{~h}$ after exercise $(\mathrm{p}<0.05)$.

Conclusion: The data reinforce the marked metabolic and hormonal demands imposed by motocross, resulting in a condition of enhanced plasma oxidative stress and damage.
\end{abstract}

M otorcycling is a motor sport modality that includes several disciplines such as speed, enduro, trial and motocross. Several particular features in off-road motocross markedly increase the physical and physiological demands for the riders. These include the irregular terrain, dirt and sharp turns that lead to the so-called "arm pump" - a muscular stress sited in the upper limbs because several enduring isometric and/or eccentric muscular contractions are necessary to absorb shocks caused by roughness and motorbike handling during the constant surface driving and landings. Given that motor sports are representative of psychoemotional stress activities, further catecholamine release is expected during this type of exercise. Notwithstanding the markedly increased number of isometric contractions, the mean oxygen consumption previously reported in motocross ${ }^{1}$ is suggestive of the increased metabolic aerobic contribution for performance. In this regard, it is tempting to hypothesise that these characteristics, isolated or in combination, may favour the generation of enhanced reactive oxygen and nitrogen species (RONS). This so-called oxidative stress results from an imbalance between production of RONS such as superoxide $\left(\mathrm{O}_{2}{ }^{-}\right)$, hydrogen peroxide $\left(\mathrm{H}_{2} \mathrm{O}_{2}\right)$, hydroxyl radical $\left(\mathrm{OH}^{-}\right)$and peroxinitrite $\left(\mathrm{ONOO}^{-}\right)$, and the ability of body antioxidant systems to counteract RONS. The consequent muscle oxidative damage, which has been widely reported after different types of physical exercise, predominantly during eccentric exercise, ${ }^{2}$ contributes to temporary loss of the exercising capacity of the muscle for force production, compromising exercising capacity.

To date and to the best of our knowledge, there has been no published study analysing the effect of motocross on oxidative stress and damage biomarkers. Thus, the purpose of this study was to examine the effect of a motocross heat on the plasma content of oxidative stress (total (TGSH), reduced (GSH) and oxidised (GSSG) glutathione and total antioxidant status (TAS)) and damage (malondialdehyde (MDA), sulphydryl and carbonyls). Levels of urine catecholamine, plasma uric acid and blood leucocyte counts were also determined.

\section{METHODS}

\section{Participants}

Ten top elite non-smoker male off-road motocross riders with international experience participated in this research after being informed about the aims and experimental protocol, and after giving written consent to participate. This protocol was approved by the ethics committee of the Faculty of Sport, University of Porto, Porto, Portugal, which followed the Declaration of Helsinki for research on humans. All subjects were previously familiarised with the settings of this study.

For 4 weeks before the data collection, participants were asked to refrain from additional vitamin dietary supplementation. The riders were instructed not to introduce appreciable deviations from their normal eating habits during this period.

The participants were tested within 1 week, and the experimental protocol included a laboratory and a field test session, separated by 3 days (fig 1).

\section{Laboratory and field evaluations}

After prediction of the percentage of body fat through skin-fold measurement, the riders performed an incremental treadmill (Quasar-Med, Nussdorf, Germany) test until voluntary exhaustion to determine maximal oxygen uptake, maximum heart rate and anaerobic ventilatory threshold (Lan). Expired respiratory gas fractions were measured using an open circuit breath-bybreath automated gas-analysis system (Cortex, Metalyzer, 3 B, Leipzig, Germany).

Heart rate was measured using a heart rate monitor (Vantage NV, Polar Electro, Kempele, Finland). Capillary blood was collected from the earlobe and immediately analysed using an electroenzymatic device (YSI 1500S, Yellow Springs, Ohio, USA).

Abbreviations: GSH, reduced glutathione; GSSG, oxidised glutathione; MDA, malondialdehyde; RONS, reactive oxygen and nitrogen species; TAS, total antioxidant status; TGSH, total glutathione 


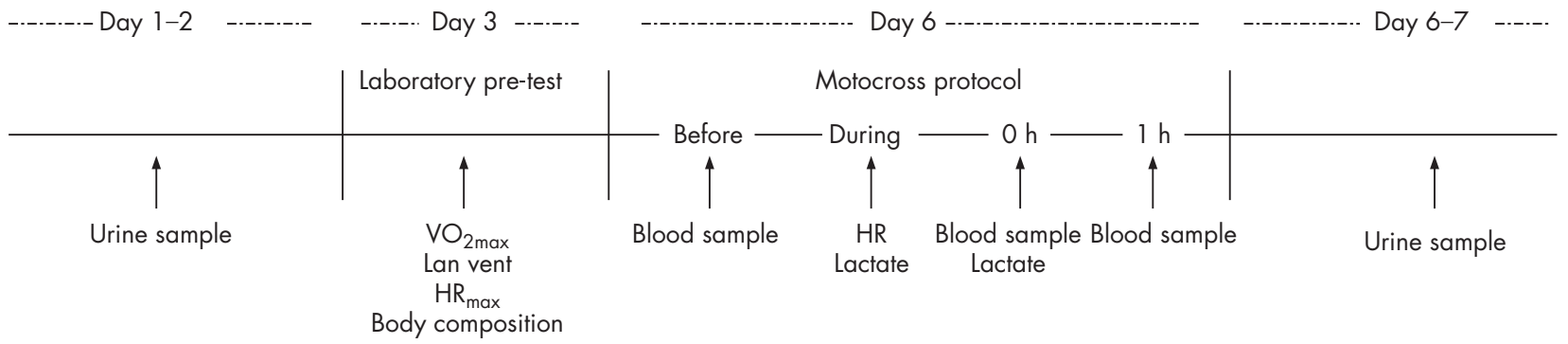

Figure 1 Scheme summarising the general lines of the experimental protocol. $\mathrm{HR}$, heart rate; Lan vent, ventilatory threshold; $\mathrm{VO}_{2}$, oxygen consumption.

Participants underwent a simulated competitive heat in a 1.5-km motocross circuit. Changes induced by race effort on biochemical markers (capillary blood lactate, urine catecholamine contents, plasma oxidative stress and damage) were analysed before, immediately after $(0 \mathrm{~h})$, and $1 \mathrm{~h}$ after the end of the race. Heart rate and blood lactate concentration were outlined throughout the heat.

\section{Catecholamine measurements}

Urine catecholamine levels were determined before and after (24 hour continuous urine sample) the heat by high-performance liquid chromatography (Waters 600 Systems, Waters Corporation, Milford, Massachusetts, USA) with electrochemical detection, using a commercial kit from Chromsystems (ref 6000, Munich, Germany). 3,4-Dihydroxybenzylamine hydrobromide (Sigma, Missouri, USA) was used as the internal standard. All steps related to the procedures of sample preparation, extraction and elution were performed according to the manufacturers' instructions.

\section{Blood lactate}

During the race, each rider was stopped for only $15 \mathrm{~s}$ at the 10th and 20th minutes to collect a 30- $\mu$ l blood sample, which was immediately analysed for lactate concentration as described. Blood lactate concentration was also measured immediately before and after the end of the race.

\section{Venous blood sampling and preparations}

Blood samples $(5 \mathrm{ml})$ were drawn three times during the experimental protocol from the antecubital vein. The first blood sample was drawn at rest and the other two at $0 \mathrm{~h}$ (immediately after the end of the race) and after $1 \mathrm{~h}$. No tourniquet constriction was used while drawing blood in order to minimise potentially enhanced oxidative stress induced by an ischaemia-reperfusion manoeuvre. All samples were taken using plastic syringes, placed in EDTA-containing tubes and were immediately centrifuged for $10 \mathrm{~min}$ at $1500 \mathrm{rpm}$. An aliquot of whole blood was separated for leucocyte counts. From the remaining blood, plasma was separated into several aliquots and rapidly frozen at $-80^{\circ} \mathrm{C}$ for later biochemical analysis of glutathione, sulphydryl, MDA, carbonyl and uric acid contents.

\section{Leucocyte cell counts}

Leucocyte count was assessed by an automatic cell counter (Horiba ABX Micros 60, ABX Diagnostics, Montpellier, France) calibrated with an ABX Minocal (ABX Diagnostics); the intraassay coefficient of variation determined on five replicates of each leucocyte measurement was $<1 \%$. Whole blood smears on glass slides (VBS 655/A Microscope, Biosigma, Venice, Italy) were used for differential analysis of white cells. Smears were stained using Wright coloring (Merck, Darmstadt, Germany) and air-dried. Cell differentials were performed using an
Olympus microscope equipped with a $\times 1000$ oil immersion lens. Specifically, the leucocyte counts, including neutrophils, eosinophils, basophils, monocytes and lymphocytes were recorded.

\section{Oxidative stress and damage markers}

Uric acid was determined by an enzymatic method at $550 \mathrm{~nm}$ using a commercial kit (Horiba ABX AllA01670, ABX Diagnostics) according to the specifications of the manufacturer.

TAS was measured spectrophotometrically at $600 \mathrm{~nm}$ using a commercial kit (Randox NX2332, Randox, Crumlin, UK).

Plasma TGSH and GSSG measurements were spectrophotometrically determined at $412 \mathrm{~nm}$ as described previously by Tietze. ${ }^{3}$ TGSH and GSSG concentrations were established on the basis of calibration curves made using commercial standards. Reduced (GSH) and percentage of oxidised glutathione (\%GSSG) were calculated as follows:

$$
\mathrm{GSH}=\mathrm{TGSH}-\mathrm{GSSG} ; \% \mathrm{GSSG}=(\mathrm{GSSG} / \mathrm{TGSH}) \times 100
$$

The plasma content of oxidative modified sulphydryl protein groups was quantified by spectrophotometric measurement at $414 \mathrm{~nm}$ according to the method proposed by $\mathrm{Hu} .{ }^{4}$

Lipid peroxidation was spectrophotometrically measured at $535 \mathrm{~nm}$ by determining the levels of lipid peroxides as the amount of thiobarbituric acid reactive substances formed, according to the method of Rohn et al, ${ }^{5}$ with some modifications.

Protein carbonyl derivatives were assayed according to the method of Robinson et al, ${ }^{6}$ with some modifications. A plasma volume containing $20 \mu \mathrm{g}$ of protein was derivatised with dinitrophenylhydrazine. After diluting the derivatised proteins in Tris-buffered saline, a volume of $100 \mathrm{ml}$ was slot-blotted into a Hybond-polyvinylidene difluoride membrane. Immunodetection of carbonyls was then performed using rabbit polyclonal antidinitrophenyl (1:2000; V0401 DakoCytomation, Copenhagen, Denmark). Bands were visualised by treating the immunoblots with enhanced chemiluminescence reagents (Amersham,

\begin{tabular}{lc}
\hline Table 1 & Characteristics of the participants \\
\hline Variables & Mean (SD) \\
\hline Age (years) & $28.3(7.9)$ \\
Mass (kg) & $71.1(7.0)$ \\
Height (cm) & $169.8(4.0)$ \\
Percentage of body fat & $14.9(3.3)$ \\
$\mathrm{HRmax}_{(\mathrm{beats} / \mathrm{min})}$ & $198.3(4.4)$ \\
$\mathrm{VO}_{2} \mathrm{max}(\mathrm{l} / \mathrm{min})$ & $3.8(0.4)$ \\
$\mathrm{VO}_{2} \mathrm{max}(\mathrm{ml} / \mathrm{kg} / \mathrm{min})$ & $53.5(3.7)$ \\
Lan vent $(\mathrm{ml} / \mathrm{kg} / \mathrm{min})$ & $42.0(5.6)$ \\
\hline
\end{tabular}

$\mathrm{HR}$, heart rate; Lan vent, ventilatory threshold; $\mathrm{VO}_{2}$, oxygen consumption. 
Table 2 Main characteristics of the race

\begin{tabular}{ll}
\hline Parameter & 30 min \\
\hline HRmax lab (beats/min) & $198.3(4.4)$ \\
HRmax field (beats/min) & $197.7(8.0)$ \\
HR average (beats/min) & $180.7(9.5)$ \\
$\% \mathrm{O}_{2}$ max & $90.8(6.8)$ \\
Lactate concentration (mmol/I) & $5.4(1.2)$ \\
\hline & \\
HRmax lab, maximum heart rate obtained in the laboratory test; \\
HRmax field, maximum heart rate obtained during the race; \\
HRmax average, average heart rate; \%VO $\mathrm{O}_{2}$ max, estimated \\
intensity of the race from the corresponding heart rate \\
determined in the lab treadmill running test and expressed in \\
$\%$ V ${ }_{2}$ max. \\
Values are mean (SD).
\end{tabular}

Pharmacia Biotech, Buckinghamshire, UK), according to the supplier's instructions, followed by exposure to $x$ ray films (Sigma, Kodak Biomax Light Film, St Louis, Missouri, USA). The films were analysed with QuantityOne Software 4.3.1 (BioRad, California, USA). Optical density results were expressed as the percentage variation of control values. Protein content was spectrophotometrically assayed using bovine serum albumin as standard according to the method of Lowry et al.

\section{Statistics}

Descriptive measures were calculated for all variables. Analysis of variance for repeated measures was used to compare biochemical variables between baseline, $0 \mathrm{~h}$ and $\mathrm{l} \mathrm{h}$. Pearson's correlation coefficient was used to analyse the intercorrelations between uric acid and TAS. SPSS V.12.0 was used for all analysis. The significance level was set at 5\%.

\section{RESULTS}

Table 1 shows the anthropometric and physiological characteristics of participants. On the day of the heat, temperature and relative humidity levels were within the range of $18-22^{\circ} \mathrm{C}$ and $60-65 \%$, respectively. Increased heart rate and estimated oxygen consumption were observed during the race (table 2 ).

Plasma carbonyl levels increased both at $0 \mathrm{~h}$ and at $\mathrm{l} \mathrm{h}$ after exercise (fig 2). Plasma lipoperoxidation, expressed as MDA level rose significantly immediately after the motocross heat and returned to initial values after $1 \mathrm{~h}(\mathrm{p}<0.05)$. Sulphydryl protein groups decreased significantly both immediately and at $1 \mathrm{~h}$ after the end of the race $(\mathrm{p}<0.05)$. Motocross race induced a significant decrease in TGSH and GSH levels, whereas GSSG and \%GSSG increased. These changes were observed both immediately and at $1 \mathrm{~h}$ after the race $(\mathrm{p}<0.05)$. A significant increase in plasma uric acid as well as in TAS from baseline to $0 \mathrm{~h}$ and $\mathrm{l} \mathrm{h}$ after motocross race was noticed (table 3 ).
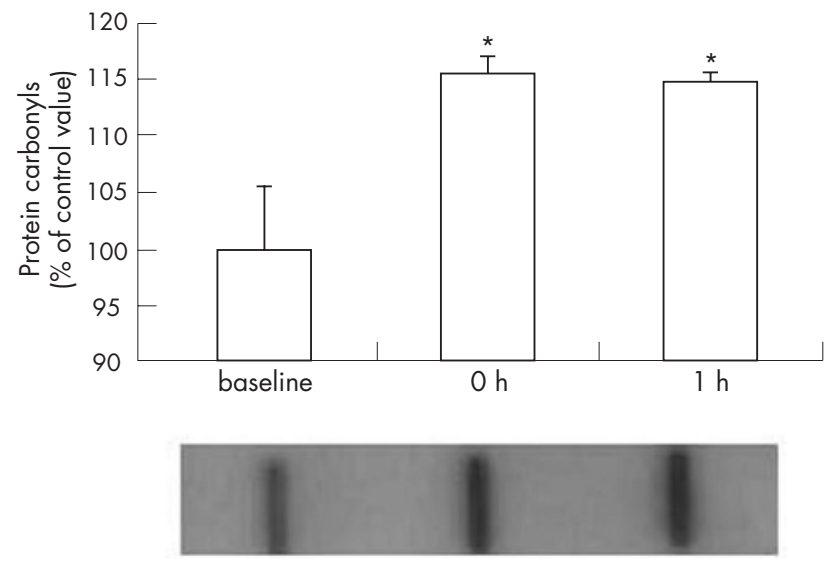

Figure 2 Effect of motocross heat on the content of plasma carbonyl derivatives obtained from eight independent participants. Immediately below the histogram, the protein carbonyl formation panel shows a representative pattern of anti-dinitrophenyl (DNP)-specific interaction with DNP for each moment as described in the Methods section. Values (mean (SEM)) are expressed as percentage of baseline. ${ }^{*} p<0.05$, baseline $v$ other moments.

Significant correlation was found between TAS and uric acid levels $(\mathrm{r}=0.72 ; \mathrm{p}<0.001)$.

Blood leucocyte and neutrophil counts were markedly higher at $0 \mathrm{~h}$ and $\mathrm{l} \mathrm{h}$ after exercise when compared with pre-exercise levels $(\mathrm{p}<0.05)$. Although lymphocyte counts rose considerably at $0 \mathrm{~h}$ after exercise, they decreased below rest values at $1 \mathrm{~h}$ after exercise (table 4).

A considerable increase in the 24-h urine catecholamine (adrenaline, noradrenaline and dopamine) concentration was found after the race compared with values obtained at rest $(\mathrm{p}<0.05$, fig 3$)$.

\section{DISCUSSION}

Specific motocross effort implies several particular acute physiological changes such as increased cardiac output and blood flow, augmented catecholamine release, high contractile isometric and eccentric demands, and importantly, relies on aerobic metabolism. Given that these are predisposing conditions for pro-oxidant redox changes in the human body, we tested the hypothesis that a simulated motocross heat induces changes in plasma markers of oxidative stress and damage. Our data showed for the first time that a single competitive heat results in an increased expression of oxidative stress and damage markers in plasma, urinary catecholamines, and considerable changes in neutrophil and lymphocyte counts.

Table 3 Plasma contents of total, reduced, oxidised (GSSG) glutathione, \%GSSG, malondialdehyde and sulphydryl protein groups, total antioxidant status and uric acid at baseline, immediately and $1 \mathrm{~h}$ after the race

\begin{tabular}{llll}
\hline Variable & Baseline & O h & l h \\
\hline TGSH (nmol/mg prot) & $0.234(0.05)$ & $0.187(0.02)^{*}$ & $0.183(0.04)^{*}$ \\
GSH (nmol/mg prot) & $0.216(0.03)$ & $0.142(0.06)^{*}$ & $0.146(0.03)^{*}$ \\
GSSG (nmol/mg prot) & $0.018(0.005)$ & $0.045(0.006)^{*}$ & $0.037(0.003)^{*}$ \\
\%GSSG & $7.82(0.54)$ & $24.95(1.02)^{*}$ & $19.99(1.05)^{*}$ \\
MDA (nmol/g prot) & $324.79(13.73)$ & $394.63(11.91)^{*}$ & $303.43(16.76)$ \\
sulphydryl (mmol/g prot) & $6.21(0.22)$ & $5.35(0.18)^{*}$ & $4.04(0.29)^{*}$ \\
TAS (mmol/l) & $1.51(0.05)$ & $1.83(0.06)^{*}$ & $1.86(0.05)^{*}$ \\
UA (mg/dl) & $6.36(0.35)$ & $7.34(0.46)^{*}$ & $7.26(0.53)^{*}$
\end{tabular}

GSH, reduced glutathione; GSSG, oxidised glutathione; MDA, malondialdehyde; TAS, total antioxidant status; TGSH, total glutathione; UA, uric acid.

Values are mean (SEM).

${ }^{*} \mathrm{p}<0.05 v$ baseline. 
Table 4 Changes in blood leucocyte, neutrophil and lymphocyte counts before, immediately and $1 \mathrm{~h}$ after simulated competitive motocross race

\begin{tabular}{|c|c|c|c|}
\hline Variable & Baseline & $\mathrm{Oh}$ & $1 \mathrm{~h}$ \\
\hline $\begin{array}{l}\text { Leucocytes } \\
\text { (10 } 10^{9} \text { blood cells } / \text { I) }\end{array}$ & $7.07(0.54)$ & $9.77(0.58)^{\star}$ & $10.27(0.85)^{*}$ \\
\hline $\begin{array}{l}\text { Neutrophils } \\
\text { (10 } 10^{9} \text { blood cells } / \text { I) }\end{array}$ & $4.31(0.51)$ & $5.41(0.60)^{*}$ & $7.82(0.79)^{*} \dagger$ \\
\hline $\begin{array}{l}\text { Lymphocytes } \\
\text { ( } 10^{9} \text { blood cells/l) }\end{array}$ & $2.02(0.09)$ & $3.41(0.26)^{*}$ & $1.68(0.07)^{*} \dagger$ \\
\hline \multicolumn{4}{|c|}{$\begin{array}{l}\text { Values are mean (SEM). } \\
{ }^{\circ} \mathrm{p}<0.05 \vee \text { baseline. } \\
\text { † } \mathrm{h} v 1 \mathrm{~h}(\mathrm{p}<0.05)\end{array}$} \\
\hline
\end{tabular}

The participants in this study were high-level professional motocross riders involved in daily specific training sessions. Several studies in the literature have reported that chronic exercise might attenuate the increased oxidative stress and damage caused by severe acute stimuli. ${ }^{8}$ This fact has been attributed to the increase in endogenous upregulation of both enzymatic and non-enzymatic antioxidants and/or a more tightly coupled electron transport system allowing fewer electrons to escape from mitochondrial electron transport chain and thus form $\mathrm{O}_{2}{ }^{-}$radicals (this for mostly aerobic exercise). Therefore, and considering the training status of the riders, a lack of changes in the markers of oxidative stress and damage was initially unlikely to exclude.

As an estimated $1-5 \%$ of the total oxygen consumption results in the formation of $\mathrm{O}_{2}{ }^{-}$, ? and given the high level of oxygen consumption accompanying a motocross heat, it is not surprising that the biomarkers of oxidative stress and damage had increased. In addition, other concurrent factors can influence cellular and blood antioxidant status. For example, stress hormones undergoing autoxidation ${ }^{10}$ and circulating neutrophil-induced oxidative burst ${ }^{11}{ }^{12}$ can contribute to the observed blood oxidative stress and damage. The influence of isometric exercise-mediating muscular ischaemia-reperfusionlike events on the formation of RONS has also been reported. ${ }^{13}$ Considering the specific physiological demands imposed by motocross, none of these potential RONS sources should be ruled out in the current study. However, it is important to note that under the technical constraints of this study, we cannot conclusively show a casual link between any of those potential sources and the increased plasma oxidative stress and damage found.

As \%GSSG is a sensitive marker of enhanced oxidative stress closely related to brisk cellular redox changes, the considerable increase from baseline to $0 \mathrm{~h}$ and $\mathrm{l} \mathrm{h}$ after exercise seems to show additional free radical production and an overwhelming of antioxidant capacity induced by the race. The increase in $\%$ GSSG observed immediately after the end of the heat suggests that during this stress period, and despite tissue GSH oxidation and compensatory GSH importation from plasma to protect cells against enhanced RONS production, hepatic exportation of GSH to circulation was insufficient to maintain its concentration. Consequently, the levels of plasma TGSH decreased, regardless of the rise observed in plasma GSSG. The increased \%GSSG was probably related to both marked plasma GSH oxidation and GSSG exportation from the tissues, owing to its toxic effect and destabilising action on cell redox status when present at high concentrations. ${ }^{14}$ Indeed, off-road motocrossinduced tissue oxidative stress seems to lead to incapacity of the liver to keep up sufficient GSH exportation to maintain the plasma levels and consequently to plasma redox status.

Surprisingly, we found that plasma TAS significantly increased after the race, which may indicate compensation in response to intense exercise. Previous studies have shown that half-marathon running by trained male runners ${ }^{15}$ and treadmill running until exhaustion ${ }^{16}$ also induced an increase in total antioxidant capacity. Considering that TAS assay only measures the antioxidant capacity of the aqueous blood compartment, which relies mostly on protein (10-28\%), uric acid (7-58\%) and ascorbic acid $(3-27 \%),{ }^{17}$ the increase in TAS observed immediately after exercise seems to reflect and/or be influenced, at least partially, by the significant increase observed in uric acid, as suggested by the increased correlation found between TAS and uric acid $(\mathrm{r}=0.72 ; \mathrm{p}<0.001)$. In fact, despite being an end product of the purine nucleotide system, uric acid scavenges $\mathrm{OH}^{-}$radicals as well, and there is evidence that it may be an important biological scavenger against free radicals in human plasma and in skeletal muscle during and after acute hard exercise. ${ }^{18}$ This well-known free radical-quenching action of uric acid might have contributed in this particular case to an attenuation of the rise in plasma oxidative damage.

During high-intensity exercise and muscle ischaemic conditions, the purine nucleotide system is extremely active, and the elimination of adenosine monophosphate causes a build-up of hypoxanthine in skeletal muscle and in plasma. Although some may be converted back to adenosine monophosphate during rest and at lower exercise intensities, hypoxanthine is also converted to uric acid, generating $\mathrm{O}_{2}{ }^{-}$. Given the lactate concentrations measured in this study after exercise (table 3 ), possible lactate-induced inhibition of renal uric acid clearance ${ }^{19}$ may also further contribute, at least partially, to the rise in uric acid after the race. The observation that plasma uric acid levels increased in response to motocross racing is consistent with the findings from other similar studies using exercise. ${ }^{18}$ In this regard, it is likely that the observed increased oxidative stress and damage during the intense exercise that off-road motocross represents might result, at least partially, from the contribution of the xanthine oxidase superoxide-generating system.

In this study, the pro-oxidative condition induced by motocross and suggested by the increase in \%GSSG was accompanied by the considerable accumulation of lipid peroxidation and protein oxidation byproducts in plasma (table 3 and fig 2, respectively). Accordingly, the race also induced a significant reduction in plasma sulphydryl residues, indicating increased disulphide linkages (-S-S-) from both proteins and GSH.

As previously described by others, ${ }^{12}{ }^{20}$ the present data showed that motocross induced a leucocytosis dependent on neutrophilia, which can be ascribed to the mobilisation of blood cells from marginal pools by haemodynamic redistribution and

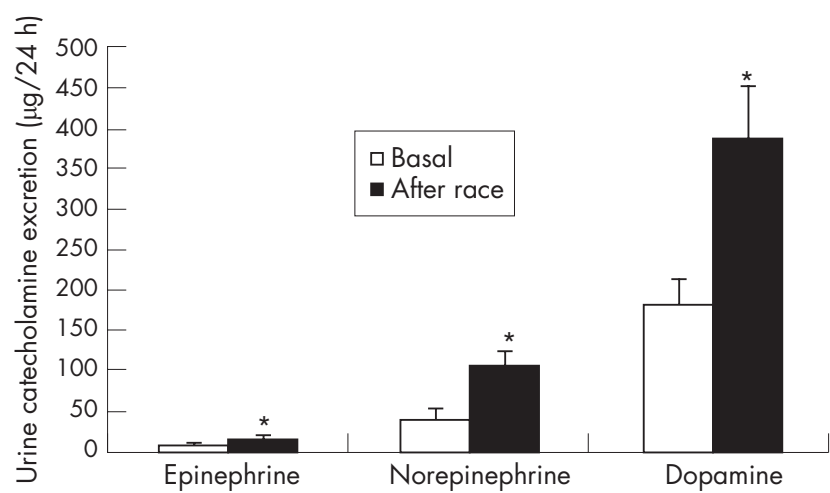

Figure 3 Mean 24-h urinary epinephrine, norepinephrine and dopamine excretion rates in riders before and after the race. Values are mean (SEM). Measurements were made at rest (throughout $24 \mathrm{~h}$ before the race) and during the immediate $24-\mathrm{h}$ period after the simulated competitive race. ${ }^{*} \mathrm{p}<0.05$, before $v$ after. 


\section{What is already known on this topic}

- Although the phenomenon of exercise-induced enhanced pro-oxidant redox changes has been widely studied, the effect of this specific exercise effort (off-road motocross race) on plasma markers of oxidative stress and oxidative damage is not known.

\section{What this study adds}

- The results of this study show that an off-road motocross heat elicited increased levels of plasma oxidative stress and damage. Although the direct effects of enhanced oxidative stress on exercise performance are inconclusive, the results of this study can be useful as a starting point for further works on the possible benefit of antioxidant supplementation on motocross performance.

augmentation that resulted from exercise-related metabolic conditions, such as enhanced catecholamine secretion imposed by motocross. Regardless of some controversy about the involvement of neutrophils in exercise-induced oxidative stress, ${ }^{21}$ previous studies using chemiluminescence techniques had shown that intense exercise was able to increase the capacity of neutrophils for RONS generation. ${ }^{11}{ }^{12}$ Nevertheless, the casual link between the increased oxidative damage and neutrophilia observed in this study should be cautiously established, as we did not measure the levels of neutrophil activation.

The data from this study reported higher lymphocyte counts immediately after exercise, and a marked lymphocytopenia during the subsequent hours after the end of exercise which is in agreement with previous studies. ${ }^{22}{ }^{23}$ Although Steensberg et $a l^{23}$ observed that, even in a study in which high levels of apoptosis-inducing factors such as cortisol and isoprostanes were generated, lymphocyte apoptosis did not contribute to post-exercise lymphocytopenia, others suggested that apoptosis may partially account for the transient loss of lymphocytes after intense exercise, with consequent immunosuppression. ${ }^{22}$ Moreover, as hormonal changes such as catecholamine overproduction during exercise have been described as responsible for inducing apoptosis, ${ }^{24}$ the increased dopamine release observed in this study might also have been a contributor.

In conclusion, the data from this study reinforce the marked metabolic and hormonal demands imposed by motocross on the riders, which results in a condition of plasma oxidative stress and damage. The specific nature and the contribution of the different RONS sources to this oxidative burst need to be clarified. Further studies in this field are needed to analyse whether more accurate and suited redox balances, obtained after antioxidant supplementation-based strategies, could be useful for the riders and their performance, particularly during intense training periods.

\section{ACKNOWLEDGEMENTS}

We thank all the riders who participated in this study. We also thank Dr Henrique Reguengo from the Department of Clinical Analysis of the
Faculty of Pharmacy, University of Porto, for his kind support in urine catecholamine assays, and Dr Rocha e Costa for his help in blood cell counts.

\section{Authors' affiliations}

António Ascensão, Rita Ferreira, José Magalhães, Research Center in Physical Activity, Health and Leisure, Porto, Portugal

Eduardo Oliveira, Victor Azevedo, José Soares, Department of Sports Biology, Faculty of Sport Sciences, University of Porto, Porto, Portugal Franklim Marques, Department of Biochemistry and Clinical Analysis, Faculty of Pharmacy, University of Porto, Porto, Portugal

Competing interests: None declared.

\section{REFERENCES}

1 Gobbi AW, Francisco RA, Tuy B, et al. Physiological characteristics of top level off-road motorcyclists. Br J Sports Med 2005;39:927-31.

2 Kuipers H. Exercise-induced muscle damage. Int J Sports Med 1994;15:132-5.

3 Tietze F. Enzymic method for quantitative determination of nanogram amounts of total and oxidized glutathione: applications to mammalian blood and other tissues. Anal Biochem 1969;27:502-22.

4 Hu M-L. Measurement of protein thiol groups and GSH in plasma. In: Parker L, eds. Methods in enzymology. San Diego: Academic Press, 1990:380-5.

5 Rohn TT, Hinds TR, Vincenzi FF. lon transport ATPases as targets for free radical damage. Protection by an aminosteroid of the $\mathrm{Ca} 2+$ pump ATPase and $\mathrm{Na}+/ \mathrm{K}+$ pump ATPase of human red blood cell membranes. Biochem Pharmacol 1993;46:525-34

6 Robinson CE, Keshavarzian A, Pasco DS, et al. Determination of protein carbonyl groups by immunoblotting. Anal Biochem 1999;266:48-57.

7 Lowry $\mathrm{OH}$, Rosenbrough N, Farr AL, et al. Protein measurement with the folin phenol reagent. J Biol Chem 1951;193:265-75.

8 Ji LL. Antioxidants and oxidative stress in exercise. Proc Soc Exp Biol Med 1999;222:283-92

9 Fridovich I. Superoxide radicals, superoxide dismutases and the aerobic lifestyle. Photochem Photobiol 1978;28:733-41.

10 Cooper CE, Vollaard NB, Choueiri T, et al. Exercise, free radicals and oxidative stress. Biochem Soc Trans 2002;30:280-5.

11 Quindry JC, Stone WL, King J, et al. The effects of acute exercise on neutrophils and plasma oxidative stress. Med Sci Sports Exerc 2003;35:1139-45.

12 Hessel E, Haberland A, Muller M, et al. Oxygen radical generation of neutrophils: a reason for oxidative stress during marathon running? Clin Chim Acta 2000;298:145-56

13 Alessio HM, Hagerman AE, Fulkerson BK, et al. Generation of reactive oxygen species after exhaustive aerobic and isometric exercise. Med Sci Sports Exerc 2000;32:1576-81.

14 Sies $\mathbf{H}$. Hydroperoxides and thiol oxidants in the study of oxidative stress in intact cells and organs. In: Sies H, eds. Oxidative stress. London: Academic Press, 1985:73-90.

15 Child RB, Wilkinson DM, Fallowfield JL, et al. Elevated serum antioxidant capacity and plasma malondialdehyde concentration in response to a simulated half-marathon run. Med Sci Sports Exerc 1998;30:1603-7.

16 Vider J, Lehtmaa J, Kullisaar T, et al. Acute immune response in respect to exercise-induced oxidative stress. Pathophysiology 2001;7:263-70.

17 Wayner DD, Burton GW, Ingold KU, et al. The relative contributions of vitamin $E$, urate, ascorbate and proteins to the total peroxyl radical-trapping antioxidant activity of human blood plasma. Biochim Biophys Acta 1987;924:408-19.

18 Tauler P, Aguilo A, Gimeno I, et al. Influence of vitamin C diet supplementation on endogenous antioxidant defences during exhaustive exercise. Pflugers Arch 2003:446:658-64.

19 Emmerson BT. Uric acid. In: Kelly WN, Weiner IM, eds. Handbook of experimental pharmacology. New York: Springer, 1978:287-324.

20 Tauler P, Aguilo A, Gimeno I, et al. Differential response of lymphocytes and neutrophils to high intensity physical activity and to vitamin $\mathrm{C}$ diet supplementation. Free Radic Res 2003;37:931-8.

21 Peake J, Quindry JC, Broeder CE. Are neutrophils really involved in exercise induced oxidative stress? Med Sci Sports Exerc 2004;36:175-6.

22 Mooren FC, Bloming $D$, Lechtermann $A$, et al. Lymphocyte apoptosis after exhaustive and moderate exercise. J Appl Physiol 2002;93:147-53.

23 Steensberg A, Morrow J, Toft AD, et al. Prolonged exercise, lymphocyte apoptosis and F2-isoprostanes. Eur J Appl Physiol 2002;87:38-42.

24 Cioca DP, Watanabe N, Isobe M. Apoptosis of peripheral blood lymphocytes is induced by catecholamines. Jpn Heart J 2000;41:385-98. 